latter two groups all but one case have clinical remission in certain days.

In the first group acute ischemic changes with massive subarachnoid hemorrhages were seen in all sections taken from many areas of brains. In the second, subarachnoid hemorrhages were rebled extensively and ischemic lesions were also seen with microscopic foci of old infarction.

IN the third, subsrachnoid hemorrhages confined to basal cisternas were present in three cases out of four. In these cases microscopical ischemic lesions were seen in the distributions of the ruptured vessels. IN addition to these old foci angionecrosis and fresh bleedings were seen in the regions of perforating arteries which were embedded in almost organized old blood clot.

\title{
f-3. Fatal Rupture of Diffuse Cerebral Telangiectasia in a Woman associated with a Spontaneous Carotid Cavernous Fistula during Cerebral Angiography
}

\author{
Tetsuya Leslie Sasabe, Yoshikazu Iwata, Katsuhito Akagi \\ and Masatsugu KoBatake \\ Department of Neurological Surgery, Osaka National Hospital \\ Fujio UEMURA \\ Department of Pathology, Osaka National Hospital
}

The pathogenesis of complications of angiography performed as preoperativerl management for a carotid cavernous fistula has not frequently been reported it which complications were emphasized to have been as severe as were those of surgery in Hamby's series. The present authors' intention is to report a case of a spontaneous carotid cavernous fistula that showed diffuse cerebral telangiertasia on the first attempt of cerebral angiography which developed a massive haemorrhage during the second attempt.

CASE REPORT: On January 21, 1968, this 28-year-old woman developed sudden left hemicrania, vomiting, and tinnitus, followed by a left pulsating $\mathrm{er}_{\text {. }}$ ophthalmos and a bruit over the head. On admission neurological examination: revealed a left cavernous carotid fistula. The first attempt of left carotid angia graphy was performed on March 12, 1968. The first film of each projection wal taken during the injection of Urografin. The arteriogram disclosed a left cartof cavernous communication through the aneurysm of the left internal carotid artert In the lateral arteriogram, the cavernous sinus, superior-, inferior ophthalmig angular-, and anterior facial veins were filled immediately via the fistula; there of only a little filling of the middle cerebral artery. On the contrary, the midd cerebral vein, the frontal ascending vein, veins of Trolard, of Rosenthal, of Labty transverse sinus, and the internal jugular vein were observed in the first film tall 
during the contrast injection. In the fronto-temporal area, diffuse cerebral telagiectasia were seen. At one week after the first attempt of angiography, serial angiography was carried out to make a circulatory state clear. No sooner had the ' $x$-rays been taken than the patient fell into coma. In spite of an intensive treatment, the patient expired 48 hours after the rupture.

Autopsy displayed marked edema on both hemispheres, and a large hematoma in the left fronto-temporal lobe. An intracavernous aneurysm was found to be isituated at the dorsolateral aspect of the left internal carotid artery. Microscopic Eexamination showed that the left internal carotid artery, at the level of immediately "proximal to the aneurysm, was characterized by partial separation of the media being edematous, intimal thickening in part, proliferation of much waved internal 3astica. At A-1 of the left anterior cerebral artery, the intima was partly thick .nd fibrotic; the internal elastica was much waved and dupricated; the media and idventitia markedly edematous and fibrotic. In the left Sylvian fissure, the "nternal elastica of the middle cerebral artery was separated; the intima, media, lind adventitia were in the same changes as A-1 of the anterior cerebral artery. 'ial vessels were found to be increased in the number and dilated, with the media nd adventitia thinner. Capillaries were also seen dilated. Extravasation, by nd large, was found in pial arterioles, penetrating arteries, and capillaries; not bserved in the venous system.

liscussion to $\mathbf{f - 3}$.

\title{
The Case of Carotid Cavernous Fistula repeated several fetal Subarachnoid Bleeding due to the Rupture of Three Intracranial Aneurysms
}

\author{
Yuji Miyazaki and Katsumi Suematsu \\ Department of Neurosurgery, Sapporo Medical College
}

A case who had three intracranial aneurysms with carotid cavernous fistula $d$ had died after several subarachnoid bleeding due to rupture of aneurysms $s$ reported.

The patient, a man of 32 , had injuried his head, had been a continuous roar the left side of his head 2 months later after head injury. He had noted irked protusion of his left eye 3 months later, under diagnosis of a carotid 'ernous fistura, the first operation (ligation of left common carotid artery) was te in 1952 and second operation (ligation of left internal and external artery) $s$ done in 1965 . In 1968 , he died after several subarachnoid bleeding.

Three aneurysms were discovered by autopsy-one at the bifrication of left erior cerebral artery and left middle cerebral artery, the other in the left 